\title{
Clinical outcomes after minimal-access surgery for recurrent lumbar disc herniation
}

\author{
Hoang Le, M.D., Faheem A. Sandhu, M.D., Ph.D., and Richard G. Fessler, M.D., Ph.D. \\ Section of Neurosurgery, University of Chicago Hospital, Chicago, Illinois
}

\begin{abstract}
Object. Experience with minimal-access surgical approaches for revision lumbar surgery has not been previously reported.

Methods. During a 7-month period, 10 consecutive patients with recurrent disc herniations underwent revision operations in which microendoscopic discectomy (MED) was performed. Perioperative data and clinical outcomes (according to Macnab criteria) were compared with those obtained in 25 consecutive patients who underwent routine singlelevel MED as well as with previously published data. Overall, outcome of the MED-treated revision group was excellent or good in $90 \%$ during a mean follow-up period of 18.5 months (minimum 12 months). Operative blood loss, duration, complications, and length of hospital stay were not significantly different between the revision and primary MED-treated groups.

Conclusions. Analysis of these early data suggests equivalent or superior results are obtained when performing MED compared with historical controls in which conventional surgery was conducted for recurrent disc surgery. The procedure appears to be a safe and effective alternative in cases in which recurrent lumbar disc herniation causes radiculopathy.
\end{abstract}

\section{KEY WORDS • microendoscopic discectomy - revision surgery • recurrent disc herniation • lumbar spine}

The authors of studies involving outcomes analysis after open lumbar microdiscectomy for primary lumbar disc herniations have reported a long-term success rate greater than $75 \%$ and a revision rate between 6 and 12\%.4,10,13,19 The advent of the MED technique allowed for a minimally invasive alternative to the traditional open microdiscectomy for decompression of the neural elements. ${ }^{5}$ Investigators in a prospective multicenter study involving MED for the treatment of lumbar disc herniations reported good/ excellent outcomes in greater than $90 \%$ of their patients in a mean follow-up period of 13 months. ${ }^{2}$ Similar results were achieved in another cohort of 150 consecutive patients in whom MED was performed for the treatment of lumbar disc herniation (mean follow-up period 12 months). ${ }^{15}$ Despite encouraging MED-related results in primary lumbar disc herniation, most surgeons hesitate to perform MED for recurrent disc herniations and revision lumbar surgery. ${ }^{6}$ Dense epidural scarring may make the procedure technically challenging and necessitate a generous exposure for complete identification of the scarred

Abbreviation used in this paper: $\mathrm{MED}=$ microendoscopic discectomy. area. ${ }^{9}$ Furthermore, the risk of increased morbidity due to revision lumbar surgery, including higher incidences of dural lacerations, nerve root injuries, and poorer long-term outcomes, may discourage most surgeons from using a minimally invasive approach to treat recurrent lumbar disc herniations.

Experiences with the MED technique for revision lumbar surgery have not been formally published. We report our clinical experience using the MED technique in the treatment of recurrent lumbar disc herniations. Its efficacy and complications in cases of recurrent disc herniation are compared between a revision and primary MED-treated group of patients.

\section{CLINICAL MATERIAL AND METHODS}

Between October 2000 and June 2001, 10 consecutive patients with recurrent same-level, ipsilateral side lumbar disc herniations underwent MED for their revision surgery. In nine open microdiscectomy was their primary surgery; in one MED was the primary technique. Data obtained in these patients are compared statistically with 25 consecutive patients who underwent surgery during the 
same period and in whom the MED system was used to treat primary single-level lumbar disc herniation; this patient group is a subset of those previously reported on for primary MED. ${ }^{15}$ All surgeries were directly supervised or performed by the senior author (R.G.F.). All patients underwent preoperative plain radiography, magnetic resonance imaging with and without $\mathrm{Gd}$, and/or computerized tomography myelography, and radiological results were consistent with clinical symptoms in all cases. Data were prospectively collected and compiled. Additional information was gathered from follow-up clinic visits and medical records.

The combined (primary- and revision-treated) patient population consisted of 17 male and 18 female patients (revision MED group six males and four females; primary MED group 12 males and 13 females). One female patient in each group was pregnant (third trimester). The mean ages in the two groups were 39 (revision) and 38 (primary) years, respectively.

Surgically treated disc levels for the primary MED group were L5-S1 (15 patients), L4-5 (eight patients), and L3-4 (two patients); for the revision MED group, there were six, four, and no patients undergoing surgery at the respective levels. All recurrent disc herniations were noted to be lateral on neuroimaging studies and clinically to produce unilateral radicular symptoms. In all cases, disc herniation was discovered at surgery and radiculopathy was not attributable to epidural fibrosis or scarring.

Surgery was performed after induction of general anesthesia with the patient placed prone and the spine flexed on a Wilson frame, except for the two pregnant patients in whom the lateral decubitus position was assumed for surgery. The surgical technique for lumbar MED has been outlined in a previous report, ${ }^{15}$ the technique for revision MED is similar, with the following caveats: Steinmann pin insertion and serial dilation are undertaken over the middle or lateral aspect of the facet joint; soft tissue is cleared from the facet in a lateromedial direction; the medial edge of the facet is defined with curette, and the plane between the dura and the medial facet is appreciated and enlarged. Typically, very little ligamentum flavum is present; however, if this is not the case, it is left in place to protect the dura mater. A medial facetectomy is completed; the ligamentum flavum, if present, is removed; the nerve root is identified and retracted; and the discectomy is completed. In experienced hands, the surgical procedure proceeds in a remarkably similar fashion to a primary MED. The minimum follow-up period in both groups was 12 months (mean 18.5 months in the revision MED group and 20 months in the primary group).

The Student t-test and chi-square test were used for statistical analysis. A probability value less than 0.05 was considered significant.

\section{RESULTS}

Data were collected prospectively, and clinical outcomes were graded using modified Macnab criteria (Table 1). ${ }^{15}$ According to these criteria, at a mean of 18.5 months postoperatively, outcome was excellent in five (50\%), good in four (40\%), fair in zero, and poor in one $(10 \%)$ of the revision MED-treated patients. Complications were
TABLE 1

Summary of modified Macnab criteria outcome

\begin{tabular}{ll}
\hline \hline Outcome & \multicolumn{1}{c}{ Description } \\
\hline excellent & $\begin{array}{l}\text { free of pain } \\
\text { no restriction of mobility } \\
\text { able to return to normal work \& activities } \\
\text { occasional nonradicular pain } \\
\text { good }\end{array}$ \\
$\begin{array}{l}\text { relief of presenting symptoms } \\
\text { able to return to modified work } \\
\text { some improved functional capacity } \\
\text { still handicapped \&/or unemployed } \\
\text { poor }\end{array}$ & $\begin{array}{l}\text { continued objective symptoms of root involvement } \\
\text { additional op intervention needed at index level } \\
\text { irrespective of repeat or length of postop period }\end{array}$ \\
\hline
\end{tabular}

few but included one intraoperative CSF leak in each group (none requiring postoperative treatment) and one recurrent disc herniation in the revision MED group. No other significant morbidity including wound infection or neural injury occurred in either group. There was also no incidence of delayed instability noted in either group at last follow-up examination.

The mean operative time in the primary and the revision MED groups was 88 and 98.5 minutes, respectively $(\mathrm{p}=$ 0.42 ). Intergroup blood loss was also not significantly different $(p=0.73)$. Length of hospital stay was 12.2 hours in the revision MED group and 10.7 hours in the primary MED group $(\mathrm{p}=0.39)$.

\section{DISCUSSION}

The clinical success rate of primary open lumbar microdiscectomy has been fairly consistent, with long-term pain relief reported in 70 to $90 \%$ of cases (although evaluation methods have varied). , $10,13,19$ Microendoscopic discectomy approaches for primary lumbar disc herniation have also yielded comparable success rates, although long-term outcome data are still forthcoming. ${ }^{1,15}$ In terms of reoperation for recurrent lumbar disc herniations, open discectomy has resulted in acceptable relief of radicular pain and overall success rates comparable with the primary surgery. ${ }^{3,7,8,17,18}$ In general, factors favoring a successful outcome after reoperation for recurrent lumbar disc herniation have included a pain-free interval longer than 6 months after the initial surgery, revision incorporating a fusion, and a definitive finding of a recurrent disc at surgery. $11,16,17$

In our preliminary experience with the MED technique for recurrent lumbar disc herniation, outcomes have been encouraging. Nine $(90 \%)$ of our 10 patients experienced relief of radicular pain during a mean follow-up period of 18 months. This result compares with relief documented in $94 \%$ of 150 patients in different series in which MED was performed to treat primary lumbar disc herniation and 96\% (of 100 patients) in another series in which the same outcome scale was used. ${ }^{6,15}$ A comparative breakdown is shown in Fig. 1. Despite similar overall successful outcomes achieved in the primary and revision MED series, a smaller percentage of patients in the revision MED group can be placed in the "excellent" category compared 


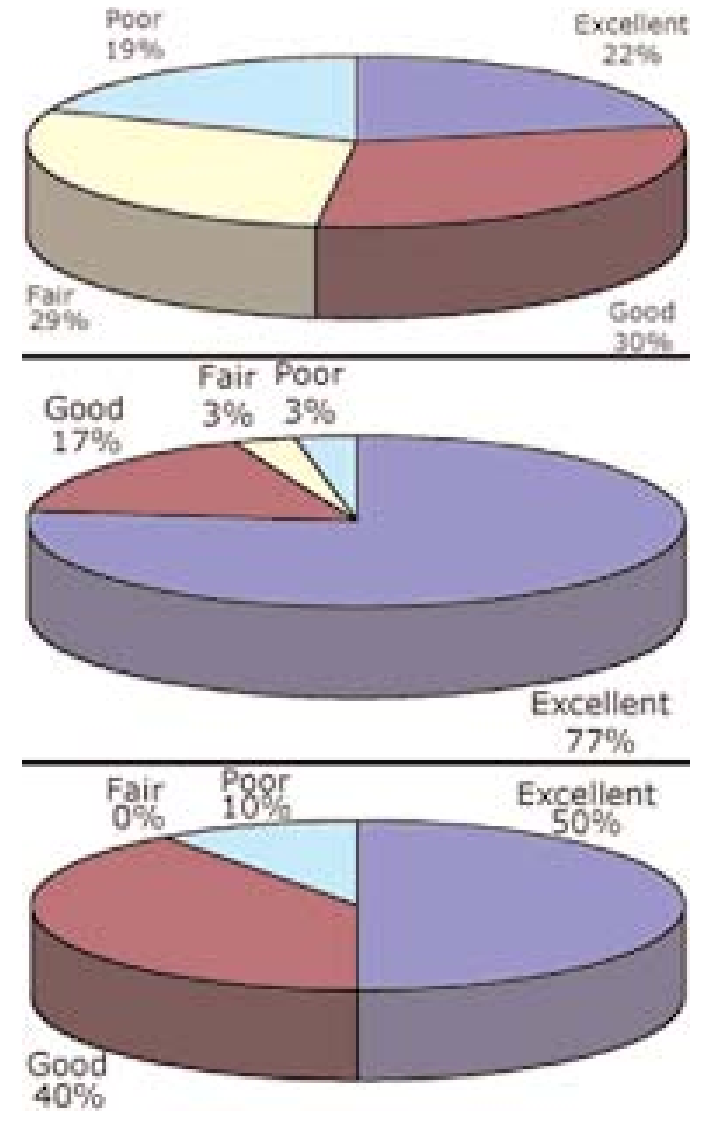

Fig. 1. Pie charts demonstrating Macnab criteria-based outcomes after open microdiscectomy for recurrent lumbar disc herniation (upper; generated from data reported by Ebeling, et al.), primary MED (center; generated from data reported by PerezCruet, et al.), and of revision MED from the present series (lower).

with the primary MED group. This trend is also observed in patients with recurrent lumbar disc herniation treated via the open microdiscectomy approach. Ebeling, et al., ${ }^{3}$ reported a success rate of $81 \%$ in their 92 patients who underwent lumbar reoperation. Further subdivision of their outcome data shows that the outcome was excellent in $22 \%$, good in $30 \%$, and satisfactory in $29 \%$. A comparative graph is shown in Fig. 1. In their series of open lumbar reoperation for radicular pain, Ozgen, et al., ${ }^{12}$ also reported a good outcome of $69 \%$ according to the Prolo Scale, which is analogous to our $89 \%$ excellent/good outcome. Other authors have also published similar clinical outcomes for open reoperative lumbar microdiscectomies. ${ }^{7,8}$

Complications in our series were few but included one dural tear in each group. Neither required further surgical intervention. No neural injury occurred. Although our follow-up period may remain short, there is no evidence in either group of delayed lumbar instability.

Although our series was limited to cases of recurrent lumbar disc herniation, minimal-access techniques are suitable for a variety of revision spinal surgeries. Residual or recurrent lateral recess stenosis could be readily approached using the MED technique following prior laminectomy or foraminotomy. Additionally, cases of failed- back syndrome requiring revision discectomy and fusion can also be treated using minimal-access techniques. In a study of 45 patients treated for single or multiple recurrent disc herniation, of which $24 \%$ required concurrent fusion, Baba, et al., ${ }^{1}$ concluded that minimal destruction of the posterior elements was crucial for improving outcomes in revision lumbar surgery. Minimal-access techniques are ideally suited for reducing destruction of posterior elements, which includes muscle, ligaments, and bone.

\section{CONCLUSIONS}

Our early experience with the MED system for recurrent lumbar disc herniations has been promising. The clinical outcomes, with a follow-up period of longer than 1 year, are comparable with previously published results after standard MED for primary lumbar disc herniation and open microdiscectomy for recurrent lumbar disc herniation. Operative time, blood loss, length of hospital stay, and complications were comparable in both primary MED- and revision MED-treated patients. We have also noticed that in reoperation in morbidly obese patients, MED has been particularly effective and perhaps preferable over the open approach. Overall, in the treatment of recurrent lumbar disc herniations, MED is a safe and effective alternative to conventional surgery.

\section{References}

1. Baba H, Chen Q, Kamitani K, et al: Revision surgery for lumbar disc herniation. An analysis of 45 patients. Int Orthop 19: 98-102, 1995

2. Brayda-Bruno M, Cinnella P: Posterior endoscopic discectomy (and other procedures). Eur Spine J 9 (Suppl 1):S24-S29, 2000

3. Ebeling U, Kalbarcyk H, Reulen HJ: Microsurgical reoperation following lumbar disc surgery. Timing, surgical findings, and outcome in 92 patients. J Neurosurg 70:397-404, 1989

4. Findlay GF, Hall BI, Musa BS, et al: A 10-year follow-up of the outcome of lumbar microdiscectomy. Spine 23:1168-1171, 1998

5. Foley K: Microendoscopic discectomy. Tech Neurosurg 3: 301-307, 1997

6. Foley KT, Smith MM, Rampersaud YR: Microendoscopic Diskectomy, in Schmidek HH (ed): Schmidek \& Sweet Operative Neurosurgical Techniques: Indications, Methods, and Results, ed 4. Philadelphia: WB Saunders, 2000, Vol 2, pp 2246-2256

7. Haglund MM, Moore AJ, Marsh H, et al: Outcome after repeat lumbar microdiscectomy. Br J Neurosurg 9:487-495, 1995

8. Herron L: Recurrent lumbar disc herniation: results of repeat laminectomy and discectomy. J Spinal Disord 7:161-166, 1994

9. Long DM: Management of Persistent Symptoms After Lumbar Disk Surgery, in Schmidek HH (ed): Schmidek \& Sweet Operative Neurosurgical Techniques: Indications, Methods, and Results, ed 4. Philadelphia: WB Saunders, 2000, Vol 2, pp 2271-2279

10. Moore AJ, Chilton JD, Uttley D: Long-term results of microlumbar discectomy. Br J Neurosurg 8:319-326, 1994

11. Osterman H, Sund R, Seitsalo S, et al: Risk of multiple reoperations after lumbar discectomy: a population-based study. Spine 28:621-627, 2003

12. Ozgen S, Naderi S, Ozek MM, et al: Findings and outcome of revision lumbar disc surgery. J Spinal Disord 12:287-292, 1999 
13. Pappas CT, Harrington T, Sonntag VK: Outcome analysis in 654 surgically treated lumbar disc herniations. Neurosurgery 30:862-866, 1992

14. Perez-Cruet MJ, Fessler RG, Perin NI: Review: complications of minimally invasive spinal surgery. Neurosurgery 51 (Suppl 5):26-36, 2002

15. Perez-Cruet MJ, Foley KT, Isaacs RE, et al: Microendoscopic lumbar discectomy: technical note. Neurosurgery 51 (Suppl 5):129-136, 2002

16. Silvers HR, Lewis PJ, Asch HL, et al: Lumbar diskectomy for recurrent disk herniation. J Spinal Disord 7:408-419, 1994

17. Suk KS, Lee HM, Moon SH, et al: Recurrent lumbar disc herniation: results of operative management. Spine 26:672-676, 2001
18. Weir BK, Jacobs GA: Reoperation rate following lumbar discectomy. An analysis of 662 lumbar discectomies. Spine 5: 366-370, 1980

19. Yorimitsu E, Chiba K, Toyama Y, et al: Long-term outcomes of standard discectomy for lumbar disc herniation: a follow-up study of more than 10 years. Spine 26:652-657, 2001

Manuscript received June 24, 2003.

Accepted in final form September 2, 2003.

Address reprint requests to: Richard Fessler, M.D., PhD., Section of Neurosurgery, University of Chicago Hospital, MC3026, 5841 South Maryland Avenue, Chicago, Illinois 60637. email: rfessler@ surgery.bsd.uchicago.edu. 